\title{
Avaliação da Competência de Estudantes de Medicina em Identificar Riscos à Segurança do Paciente através de Simulação
}

\author{
Evaluation of the Competence of Medical \\ Students in Identifying Risks to the Safety of \\ the Patient through Simulation
}

Carlos Vinicius Pacheco dos Santos Guaranal Edvaldo da Silva Souza ${ }^{I \oplus}$ Victor de Souza Dias Eliana Valentim ${ }^{1}$

\section{PALAVRAS-CHAVE}

- Segurança do Paciente.

- Treinamento por Simulação.

- Estudantes de Medicina.

- Educação Médica.

\section{RESUMO}

Introdução: A segurança do paciente (SP) está entre os principais temas discutidos pelos profissionais da área de saúde em todo o mundo. No Brasil, em 2016, ocorreram mais de 302 mil mortes por eventos adversos evitáveis e 70\% eram de causas preveníveis, que custaram mais de 10,9 bilhões de reais. Objetivo: Avaliar os conhecimentos, habilidades e atitudes de estudantes do quarto e oitavo períodos do curso de Medicina sobre segurança do paciente por meio de simulação. Método: Foi realizado um estudo transversal. A coleta de dados foi feita por meio de dois instrumentos, um com o perfil sociodemográfico e outro a ser preenchido durante a simulação. A simulação ocorreu numa sala de laboratório preparada para reproduzir um cenário hospitalar. Foram planejados pelos pesquisadores seis riscos a serem identificados no cenário durante o tempo de sete minutos. Os dados foram analisados com uso do Epi-Info 7.1. A pesquisa foi aprovada pelo Comitê de Ética da instituição. Resultado: Participaram do estudo 42 estudantes, sendo 24 do quarto período e 18 do oitavo. Nenhum estudante identificou todos os seis riscos. No geral, os alunos apresentaram baixo desempenho, tendo identificado em média de dois a três riscos à segurança do paciente. O risco de administração de medicação equivocada foi percebido por 34 estudantes (81\%); a falta de identificação por 15 (36\%); o risco de quedas por 11 (26\%); problemas de higienização das mãos por 6 (14\%); o risco de procedimentos cirúrgicos equivocados por 3 (7\%) e o risco de lesões por pressão não foi identificado por nenhum estudante. Três riscos adicionais não planejados pelos pesquisadores foram identificados pelos estudantes, com predomínio de identificação de dois riscos por estudantes do oitavo período. Conclusão: Os estudantes apresentaram pouca competência em identificar riscos à segurança do paciente. Contudo, demonstraram melhor desempenho ao longo do curso, revelando quão importante é trabalhar a segurança do paciente já nos primeiros anos de graduação. 


\section{KEY-WORDS}

- Patient Safety.

- Simulation Training.

- Students, Medical.

- Education, Medical.

\begin{abstract}
Introduction: Patient safety (PS) is among the main topics discussed by healthcare professionals worldwide. In Brazil, more than 302,000 deaths from preventable adverse events were reported in 2016, and 70\% had preventable causes that cost more than 10.9 billion reais. Objective: To evaluate the knowledge, skills and attitudes, through simulation of students of the medical course of the $4^{\text {th }}$ and $8^{\text {th }}$ semesters regarding the subject of patient safety. Methods: A cross-sectional study was conducted. The data was collected through two instruments, one to ascertain the sociodemographic profile and the other to be completed during the simulation. The simulation took place in a laboratory room prepared to reproduce a hospital scenario. The researchers planned six risks to be identified in the scenario with a duration of 7 minutes. All data were analyzed by EPI-INFO 7.1. The research was approved by an ethics committee. Results: A total of 42 students participated in the study, 24 and 18 of the $4^{\text {th }}$ and $8^{\text {th }}$ periods, respectively. No student has identified all six risks. Overall, students performed poorly, identifying on average two to three risks to patient safety. The risk of administration of wrong medication was perceived by 34 (81\%); lack of identification by 15 (36\%); risk of falling by 11 (26\%); problems with hand hygiene by 6 (14\%); risk of wrong surgical procedures by $3(7 \%)$ and risk of pressure ulcers was not identified by any student. Three additional risks not planned by the researchers were identified by the students, especially two risks identified by $8^{\text {th }}$-period students. Conclusion: Students displayed little ability to identify patient safety risks. However, they demonstrated better performance throughout the course, revealing how important it is to work on patient safety in the early years of undergraduate training.
\end{abstract}

Recebido em: 9/4/19

Aceito em: 8/6/19

\section{INTRODUÇÃO}

A segurança do paciente (SP) reflete as atitudes dos profissionais de saúde durante a sua assistência com o objetivo de prevenir eventuais falhas antes que elas causem danos ao paciente. A preocupação com a SP data de 1855, quando Florence Nightingale, uma enfermeira britânica, propôs que a higiene dos hospitais impactaria diretamente a assistência prestada a seus pacientes. Em 1910, um cirurgião americano, Ernest Codman, criou os "padrões mínimos", que visavam à melhoria constante dos serviços de saúde. Em 1999, o Instituto de Medicina divulgou o relatório "Errar É Humano", que, além de apontar as falhas dos serviços de saúde, colocou este tema na pauta da Organização Mundial da Saúde (OMS) e das políticas de saúde de diversos países. No Brasil, em 1994, surgiu o programa de acreditação para melhorar a qualidade hospitalar. Em 2011, o Ministério da Saúde (MS) divulgou um programa em que remunera os municípios e os seus serviços de saúde de acordo com o seu desempenho na qualidade da saúde prestada.

No Brasil, segundo a Agência Nacional de Vigilância Sanitária, segurança do paciente é a redução a um valor mínimo aceitável do risco de dano causado desnecessariamente na assistência prestada ao indivíduo. As situações em que há falha no cuidar do paciente são denominadas "incidentes" e não necessariamente cursam com danos à saúde. As situações que resultam em dano à saúde são chamadas "evento adverso" (EA) e podem expressar um dano temporário ou permanente ao bem-estar do paciente ou até mesmo a sua morte.

Uma pesquisa feita pelo Institute for Healthcare Improvement, realizada entre 2008 e 2011 nos Estados Unidos da América (EUA), mostrou que o número de eventos adversos graves variou de $14 \%$ a $21 \%$, o que, em números absolutos, representa um total de 210 mil mortes, em vez dos 3,7\% encontrados em pesquisa anterior feita em 1999. No Brasil, em 2016, uma pesquisa similar, realizada com base no volume de internações do Sistema Único de Saúde (SUS) e da Associação Nacional dos Hospitais Privados, concluiu que ocorreram mais de 302 mil mortes por ano por EA evitáveis.

De acordo com dados do Center for Disease Control and Prevention, dez em cada cem pacientes hospitalizados em países em desenvolvimento adquirem ao menos uma Infecção Relacionada à Assistência à Saúde (Iras), o que faz aumentar ainda mais os números da mortalidade e os gastos dos sistemas de saúde. No entanto, essa é uma realidade fácil de mudar, tendo em vista que, quando as unidades de assistência à saúde e seus multiprofissionais têm conhecimento do problema e passam a adotar medidas profiláticas, há uma redução 
de até $70 \%$ de algumas Iras, a exemplo das infecções com porta de entrada sanguínea.

Esses dados revelam a importância da abordagem da SP durante o curso de graduação dos estudantes de saúde, pois, embora a equipe interprofissional seja fundamental para eliminar os riscos que possam resultar em eventos adversos para os pacientes, a educação formal dos estudantes pode não prepará-los adequadamente para esse papel, como mostra um estudo da Universidade de Duke nos EUA. Informações sobre a implementação de protocolos de cirurgia segura, prevenção de quedas, lesões por pressão e administração de medicamentos de forma equivocada, quando fornecidas desde cedo durante o processo de formação do graduando, colaboram para a promoção de conhecimentos, habilidades e atitudes a respeito da segurança do paciente. Além disso, elucidam para o estudante a importância da temática para sua formação e, consequentemente, para a melhora da assistência prestada ao paciente.

No Brasil, diversas estratégias são utilizadas para a implementação da Política Nacional de Segurança do Paciente (PNSP), entre elas: a produção e o incentivo à adesão de protocolos, guias e manuais sobre SP; a qualificação e a capacitação de profissionais, gestores e usuários de saúde por meio de publicações do tema SP; a criação de sistemas de vigilância e monitoramento de incidentes na assistência à saúde, garantindo a contrarreferência à unidade que notificou o ocorrido. Além disso, ainda é necessária a cultura da segurança, ou seja, um conjunto de comportamentos e atitudes em favor da saúde, substituindo a penalidade pela oportunidade de aprender com os erros e assim poder oferecer melhor assistência de saúde.

No entanto, atualmente, percebe-se que muitos estudantes não recebem essas informações na graduação de forma suficiente para desenvolver as habilidades necessárias para cumprir suas responsabilidades com a segurança dos pacientes, sendo esses treinamentos oferecidos, geralmente, na pós-graduação. As escolas de Medicina ainda mantêm seu ensino voltado ao diagnóstico e tratamento das doenças, mostrando-se precário o tempo destinado ao ensino da segurança do paciente e aos sistemas de análise de erro. Por isso, há uma preocupação dos educadores de medicina em estabelecer alguns pontos de melhoria a serem abordados no ensino das escolas médicas para os cuidados com a saúde e SP, como prestação de cuidados centrados no doente, comunicação eficaz com a equipe, o paciente e seus familiares, consciência em relação ao possível erro médico, prevenção, gestão e divulgação dos erros e motivação do estudante para aprendizagem do conteúdo.

Diante dessa realidade, as Diretrizes Curriculares Nacionais (DCN) do Curso de Graduação de Medicina (2014) es- tabeleceram a prática do ensino de segurança em saúde nas escolas de Medicina, a qual inclui, entre outros pontos, a segurança na realização de procedimentos e processos e a privacidade dos usuários sobre os cuidados médicos de modo a evitar danos e efeitos adversos. Isto sempre levando em conta as melhores evidências, protocolos e diretrizes cientificamente reconhecidos para sua conduta. Além disso, as DCN orientam que se estimule o domínio de pelo menos uma língua estrangeira, como forma de interagir com equipes de outros países e participar de congressos, a fim de se manter atualizado no avanço da medicina. A implementação dessas diretrizes favorece que o estudante, durante o período acadêmico, desenvolva uma nova perspectiva de construção de conhecimento, competências e habilidades diversas de modo a se tornar um profissional crítico e reflexivo, sempre visando ao bem-estar de seus pacientes.

Para que isto ocorra, a Organização Mundial da Saúde (OMS) criou, em 2011, um guia curricular para facilitar a implementação do tema SP em instituições de ensino de todo o mundo. Esse guia é dividido em duas partes: a primeira é um guia do professor, visando capacitá-lo, tendo em vista que a SP é uma disciplina nova e que muitos docentes não sabem como abordá-la; a segunda parte é um programa vasto sobre o tema SP, bem subdividido, com o intuito de gerar conhecimento e habilidades aos estudantes da área de saúde para que possam estar capacitados nos mais variados ambientes da prática clínica.

Um estudo realizado na Universidade de Chicago (EUA) em 2015 descreveu a viabilidade de implementar um exercício simulado de SP a fim de avaliar e comparar as habilidades de estudantes de Medicina do terceiro e sexto ano de curso em termos de sua capacidade de identificar corretamente os erros a que os pacientes estão sujeitos durante a hospitalização. Dessa forma, foi criado um quarto de hospital onde um manequim simulava o paciente, e o estudante deveria listar em seu checklist os agravos que aquele paciente poderia sofrer. Resultado: alguns erros, como o risco de queda, foram identificados pela maioria dos estudantes (83\%), mas outros erros, como o risco de lesão de pressão, foram pouco percebidos pelos estudantes (5\%). Assim, o exercício mostrou-se factível e efetivo como forma de introduzir o conteúdo SP para os estudantes, visto que ambos os grupos de estudantes tiveram dificuldades em identificar os erros da hospitalização.

Estas estratégias têm como objetivo inserir e avaliar os aspectos que envolvem a segurança do paciente, como forma de alcançar saberes e habilidades específicos ainda durante a formação médica, independentemente da formação técnica que se deseje dar. Essa construção do conhecimento precisa 
estar presente em abordagens teóricas, clínicas e práticas, sendo, assim, constante no desenvolvimento dos estudantes em seus diversos cenários. No entanto, ainda não está claro de que maneira ocorre a formação e a avaliação dos estudantes de Medicina quanto ao tema segurança do paciente.

Desse modo, este estudo objetiva avaliar, por meio de simulação, os conhecimentos, habilidades e atitudes de estudantes do curso de Medicina do quarto ao oitavo período de formação acadêmica quanto ao tema segurança do paciente.

\section{MÉTODO}

Foi realizado um estudo analítico do tipo transversal. O estudo foi desenvolvido na sala do laboratório "enfermaria e UTI" da Faculdade Pernambucana de Saúde (FPS), localizada em Recife (PE), no período de agosto de 2017 a julho de 2018. A população foi composta por 42 estudantes, sendo 24 do quarto período e 18 do oitavo período. Os estudantes foram selecionados de maneira probabilística e aleatória, e todos tinham a mesma chance de serem escolhidos. Entre os estudantes elegíveis, cem demonstraram interesse em participar do estudo. Foi realizado um sorteio para estudantes do quarto e oitavo período, no qual todos os alunos tiveram a mesma chance de serem selecionados. Após essa etapa, eles receberam informações referentes à pesquisa. Foi explicitado que o objetivo da pesquisa seria realizar uma simulação acerca de um assunto comum na área de saúde, porém foi omitido, inclusive no Termo de Consentimento Livre e Esclarecido (TCLE), que seria uma simulação para avaliar a segurança do paciente, a fim de evitar vieses. Após esta explicação, os estudantes que concordaram assinaram o TCLE.

Foi definido como critério de inclusão que os estudantes estivessem devidamente matriculados no quarto e oitavo períodos do curso de Medicina da FPS. Já os critérios de exclusão foram: estudantes menores de 18 anos, estudantes que estivessem cursando ou que cursaram outros cursos na área de saúde e estudantes oriundos de outro curso de Medicina, isto é, transferidos. Dos pré-selecionados, 12 não participaram, pois preenchiam os critérios de exclusão, e 46 não tinham disponibilidade de horário. Ao final, 42 estudantes realizaram a simulação. As variáveis estudadas foram idade, sexo, período, moradia com os familiares, trabalho, escolaridade dos pais, renda familiar aproximada mensal, número de pessoas que residiam na mesma casa, horas de estudo semanal para assuntos acadêmicos, horas semanais na internet para assuntos acadêmicos, atividades extracurriculares (monitoria, pesquisa, projetos de extensão e estágios), uso de medicamentos psicoativos.

A coleta de dados foi realizada por meio de questionários, entregues presencialmente aos alunos do estudo e recolhidos após preenchimento pelos participantes da pesquisa, isto é, após o término da simulação que realizaram. Cada questionário foi composto por duas partes: a primeira era referente ao perfil sociodemográfico e ao conhecimento das características individuais dos participantes (anonimato facultativo); a segunda parte foi respondida no momento da simulação (identificação necessária caso o participante quisesse feedback).

A simulação foi realizada no laboratório da FPS, onde foram utilizados dois casos clínicos de pacientes do sexo masculino que se encontravam hospitalizados na mesma sala com doenças infectocontagiosas. Tudo se passou num cenário especialmente preparado para a realização da simulação, que continha dois manequins que representavam os pacientes internados; um dispensador de álcool gel vazio; uma pia de torneira com água, mas sem sabão; os leitos não foram identificados (sendo os pacientes identificados apenas por meio das respectivas pulseiras). Ao entrarem no laboratório da simulação, os estudantes foram avisados de que no ambiente haveria seis riscos à segurança dos pacientes, de acordo com as medidas preconizadas pelo Ministério da Saúde/OMS, a serem identificados e que eles teriam sete minutos para identificar e anotar esses riscos. Os riscos que deveriam ser identificados eram: administração segura de medicação, não identificação do paciente, ausência de prevenção de queda, ausência de material para higienização das mãos, ausência de procedimento cirúrgico seguro e o risco de lesão por pressão.

Os estudantes que optaram por receber feedback do seu desempenho se identificaram, através do e-mail, na folha usada para avaliação dos riscos durante a simulação. Eles somente obtiveram tal feedback após a análise dos resultados obtidos pela simulação, a fim de mostrar seu desempenho comparado ao dos demais. Todo o processo foi supervisionado pelos pesquisadores responsáveis pela pesquisa.

O programa utilizado para obtenção dos cálculos estatísticos foi o Epi-Info 7.1. Para as variáveis categóricas foram utilizadas frequências absolutas e percentuais. Para as variáveis contínuas foram usadas as medidas de tendência central e dispersão (média/desvio padrão, mediana/interquartil). Ao final, os dados foram apresentados em tabelas com as variáveis encontradas. O estudo foi aprovado pelo Comitê de Ética em Pesquisa da FPS sob o número CAAE 70038517.9.0000.5569.

\section{RESULTADOS}

Quarenta e dois estudantes realizaram a atividade de simulação, segundo os critérios de inclusão. Em relação às variáveis sociodemográficas (Tabela 1), 32 (76,2\%) estudantes eram do sexo feminino, $24(57,1 \%)$ estudantes eram do quarto período e $18(42,9 \%)$ do oitavo período. A média de idade foi de 21,7 (DP 
$\pm 2,4)$ anos. Trinta e três alunos $(78,6 \%)$ referiram ter os pais com ensino superior completo; 6 (14,3\%) moravam sozinhos; $2(4,8 \%)$ trabalhavam. Quanto à renda mensal familiar, apenas $15(35,7 \%)$ estudantes declararam o valor da renda, sendo a mediana de renda per capita R\$ 2.500,00 (IQ25: 1.533,00; IQ75: $5.000,00)$. Em relação ao uso de medicação, 7 (16,6\%) referiram uso atual ou anterior para ansiedade, 5 (11,9\%) referiram uso atual ou anterior para depressão e $1(2,4 \%)$ referiu uso atual ou anterior para transtorno de déficit de atenção e hiperatividade.

De acordo com a distribuição das horas semanais de estudo e outras atividades extra-acadêmicas (Tabela 2), a média das horas semanais de estudo para fins acadêmicos foi de 16,7 $(\mathrm{DP} \pm 7,4)$ horas; a média semanal do uso de internet para fins acadêmicos foi de 7,8 (DP $\pm 5,6)$ horas. Em relação a outras atividades, 16 (38\%) estudantes fizeram iniciação científica e possuíam a mediana semanal das horas dessa atividade de 4,0 (IQ25: 4,0; IQ75: 7,0) horas; entre os $5(11,9 \%)$ estudantes que participaram de projetos de extensão, a média semanal das horas foi 9,2 ( $\mathrm{DP} \pm 8,6)$ horas; entre os $8(19,0 \%)$ estudantes que fizeram estágio extracurricular, a média semanal das horas foi de 11,3 (DP $\pm 6,1$ ) horas; e entre os 13 estudantes que fizeram monitoria, a média semanal foi de 4,7 (DP $\pm 3,8)$ horas.

Em relação à atividade de simulação (Tabela 3), dos 42 estudantes participantes, nenhum deles identificou todos os seis riscos planejados para serem detectados na simulação. De forma geral, os estudantes identificaram menos da metade dos riscos planejados pelos pesquisadores. Nenhum estudante identificou o risco relacionado à prevenção de lesões por pressão. Trinta e quatro (81\%) identificaram na simulação o risco correspondente à administração segura de medicação; 15 (36\%), o risco correspondente à identificação do paciente; 11 (26\%), o risco correspondente à prevenção de quedas; 6 (14\%), o risco correspondente à higienização das mãos; e 3 (7\%), o risco correspondente à cirurgia segura.

Por outro lado, três riscos adicionais, não planejados pelos pesquisadores, foram identificados pelos estudantes (Tabela 3). O primeiro risco, correspondente ao isolamento dos pacientes, foi identificado por 24 (57\%) estudantes. Comparando

TABELA 1

Distribuição sociodemográfica dos estudantes de Medicina de acordo com o período cursado na FPS, em 2017

\begin{tabular}{|c|c|c|c|c|}
\hline Variáveis sociodemográficas & Todos & $4^{\circ}$ período & $8^{\circ}$ período & Valor de $p$ \\
\hline Idade (x, DP) & $\begin{array}{c}21,7 \\
(\mathrm{DP} \pm 2,4)\end{array}$ & $\begin{array}{c}20,6 \\
(\mathrm{DP} \pm 1,7)\end{array}$ & $\begin{array}{c}23,2 \\
(\mathrm{DP} \pm 2,4)\end{array}$ & $<0,001$ \\
\hline Número de estudantes (n,\%) & $42(100 \%)$ & $24(57 \%)$ & $18(43 \%)$ & 0,369 \\
\hline Renda per capita $(\mathrm{R} \$)$ & $3.803,00$ & $4.768,00$ & $2.699,00$ & 0,310 \\
\hline Pais com ensino superior completo $(\mathrm{n}, \%)$ & $33(79 \%)$ & $19(45 \%)$ & $14(33 \%)$ & 0,431 \\
\hline Moram sozinhos (n,\%) & $6(14 \%)$ & $3(7 \%)$ & $3(7 \%)$ & 0,999 \\
\hline Usam ou já usaram medicamentos para ansiedade $(n, \%)$ & $7(17 \%)$ & $4(10 \%)$ & $3(7 \%)$ & 0,733 \\
\hline Usam ou já usaram medicamentos para depressão (n,\%) & $5(12 \%)$ & $3(7 \%)$ & $2(5 \%)$ & 0,789 \\
\hline Usam ou já usaram medicamentos para TDAH* $(n, \%)$ & $1(2,5 \%)$ & $0(0 \%)$ & $1(2,5 \%)$ & 0,436 \\
\hline
\end{tabular}

*TDAH = Transtorno de déficit de atenção e hiperatividade.

TABELA 2

Distribuição de média de horas semanais de estudos dedicados às atividades acadêmicas e extraacadêmicas dos estudantes de Medicina de acordo com o período cursado na FPS, em 2017

\begin{tabular}{|c|c|c|c|c|}
\hline Média das horas semanais & $\begin{array}{l}\text { Todos } \\
\text { X (DP) }\end{array}$ & $\begin{array}{c}4^{\circ} \text { período } \\
\text { X (DP)* }\end{array}$ & $\begin{array}{c}8^{-0} \text { período } \\
\text { X (DP) }\end{array}$ & Valor de $p$ \\
\hline Estudos acadêmicos & $16,7( \pm 7,4)$ & $18,6( \pm 5,5)$ & $13,8( \pm 8,9)$ & 0,085 \\
\hline Uso de internet para fins acadêmicos & $7,8( \pm 5,6)$ & $8,7( \pm 5,1)$ & $6,5( \pm 6,2)$ & 0,311 \\
\hline Projeto de extensão & $9,2( \pm 8,6)$ & $5( \pm 5,6)$ & $12( \pm 10,1)$ & 0,396 \\
\hline Iniciação científica & $7,3( \pm 9,2)$ & $3,6( \pm 1,2)$ & $11( \pm 12,2)$ & 0,131 \\
\hline
\end{tabular}

* X (DP): valor da média aritmética com o valor do desvio padrão.

** Valor referente a dois participantes. 


\begin{tabular}{|c|c|c|c|c|c|}
\hline $\begin{array}{r}\text { Distribuição da } \\
\text { Medici }\end{array}$ & $\begin{array}{l}\text { cação de } \\
\text { cordo con }\end{array}$ & $\begin{array}{l}\text { ELA } 3 \\
\text { no cenáric } \\
\text { ríodo curs: }\end{array}$ & $\begin{array}{l}\text { lado dos es } \\
\text { FPS, em } 2\end{array}$ & ntes de & \\
\hline Riscos avaliados & Todos & $4^{\circ}$ período & $8^{\mathrm{o}}$ período & OR $(\mathrm{IC})^{* *}$ & Valor de $p$ \\
\hline Administração segura de medicação (n, \%) & $34(81 \%)$ & $18(75 \%)$ & $16(89 \%)$ & $\begin{array}{c}2,7 \\
(2,7 ; 2,9)\end{array}$ & 0,256 \\
\hline Identificação do paciente (n, \%) & $15(36 \%)$ & $7(29 \%)$ & $8(44 \%)$ & $\begin{array}{c}1,9 \\
(1,0 ; 1,8)\end{array}$ & 0,306 \\
\hline Prevenção de queda (n, \%) & $11(26 \%)$ & $4(17 \%)$ & $7(39 \%)$ & $\begin{array}{c}3,2 \\
(2,4 ; 2,9)\end{array}$ & 0,105 \\
\hline Higienização das mãos (n, \%) & $6(14 \%)$ & $4(17 \%)$ & $2(11 \%)$ & $\begin{array}{c}0,6 \\
(0,1 ; 0,5)\end{array}$ & 0,610 \\
\hline Cirurgia segura $(n, \%)$ & $3(7 \%)$ & $1(4 \%)$ & $2(11 \%)$ & $\begin{array}{c}2,9 \\
(1,7 ; 2,2)\end{array}$ & 0,387 \\
\hline Isolamento* $(\mathrm{n}, \%)$ & $24(57 \%)$ & $10(42 \%)$ & $14(78 \%)$ & $\begin{array}{c}4,9 \\
(4,8 ; 5,2)\end{array}$ & 0,019 \\
\hline Distância entre os leitos* (n, \%) & $5(12 \%)$ & $5(21 \%)$ & 0 & - & 0,039 \\
\hline Equipamento de Proteção Individual* (n, \%) & $5(12 \%)$ & $1(4 \%)$ & $4(22 \%)$ & $\begin{array}{c}6,6 \\
(5,4 ; 6,0)\end{array}$ & 0,073 \\
\hline
\end{tabular}

* Riscos adicionais não planejados pelos pesquisadores.

** OR (IC): Odds Ratio (Intervalo de Confiança).

a identificação de acordo com o período, 10 (42\%) estudantes do quarto período e 14 (78\%) estudantes do oitavo período identificaram este risco, demonstrando que os estudantes do oitavo período identificam mais este risco, com diferença estatística ( $p=0,019)$. Contudo, quanto ao segundo risco, a insuficiente distância entre os leitos, somente os estudantes do quarto período o indicaram. Já o terceiro risco, correspondente ao uso de equipamentos de proteção individual, foi identificado por 5 (12\%) estudantes.

A análise univariada, considerando as variáveis sociodemográficas como de exposição e os períodos do curso como desfecho, revelou não haver diferença estatística significativa, com exceção da idade dos participantes $(p<0,001)$, em que os estudantes do oitavo período tiveram uma média de idade maior que os do quarto período. Em relação à média das horas semanais de atividades acadêmicas e extra-acadêmicas, não houve diferença estatística significativa entre os períodos, com exceção das horas semanais dedicadas à monitoria $(p<0,001)$. Entre os riscos analisados durante a simulação, não houve diferença estatística na identificação dos riscos planejados pelos pesquisadores comparando-se os estudantes do quarto e oitavo períodos. Contudo, entre os três riscos adicionais identificados e não planejados, houve diferença estatística significativa quanto à identificação do risco de isolamento dos pacientes segundo os períodos dos participantes: os estudantes do oitavo período identificaram mais a necessidade de isolamento e Equipamento de Proteção Individual (EPI) com Odds Ratio (OR) 4,9 e Intervalo de Confiança (IC) 4,7-5,1 ( $p=0,019)$ e distância entre os leitos $(p=0,039)$, ao passo que os estudantes do quarto período identificaram mais a distância entre os leitos, não tendo sido possível calcular OR e IC.

\section{DISCUSSÃO}

O ensino sobre segurança do paciente nos currículos dos cursos de saúde mostra-se fragmentado, carecendo de aprofundamento e amplitude conceitual, como recomenda o guia da Organização Mundial da Saúde. Cada categoria profissional valoriza os aspectos específicos para a sua formação considerados mais pertinentes ou que se mostrem mais importantes, como evidenciou um estudo da Universidade Federal de São Paulo que avaliou as propostas pedagógicas de seus cursos da área de saúde. Nosso estudo demonstrou que as diretrizes curriculares e os estudantes de Medicina ainda parecem valorizar mais os aspectos relacionados à doença do que aspectos mais preventivos de segurança do paciente.

Em relação ao perfil dos participantes do estudo, há predomínio do sexo feminino, com média de idade de 21,7 anos. Poucos estudantes residem sozinhos ou trabalham, e a maioria dos pais tem ensino superior completo e detém renda per capita de $\mathrm{R} \$ 3.800,00$. Esse perfil é condizente com o perfil dos estudantes de Medicina de outros estados da federação e países.

Há uma tendência entre os estudantes de Medicina ao uso de medicamentos para ansiedade, depressão e transtorno de déficit de atenção e hiperatividade. No entanto, todos os números encontrados em nosso estudo foram menores que a prevalência encontrada (ansiedade 33\%, depressão 31\% e TDAH $8 \%$ ) em revisão sistêmica com metanálise que avaliou a prevalência de transtornos psiquiátricos entre os estudantes 
de Medicina do Brasil e numa pesquisa realizada pelo Instituto de Psiquiatria da Universidade Federal do Rio de Janeiro.

Neste estudo, a média de horas semanal dedicada ao estudo em atividades acadêmicas extraclasse foi de 17 horas, além do uso de mais 8 horas de internet para o mesmo fim, totalizando uma média de 25 horas semanais dedicadas ao estudo em atividades acadêmicas extraclasse. Essa média é maior que a encontrada pelo Instituto de Pesquisa Econômica Aplicada (Ipea), que avaliou as atividades acadêmicas extraclasse de estudantes universitários brasileiros oriundos de duas instituições públicas e quatro privadas. Contudo, sabe-se que o curso de Medicina, por ser de dedicação exclusiva, geralmente requer mais horas de estudo.

Quando os alunos foram avaliados de acordo com atividades extra-acadêmicas (monitoria, estágios, iniciação cientifica, extensão), essas médias foram menores que as encontradas para o estudo extraclasse, e apenas o item monitoria apresentou diferença estatística entre os grupos de estudantes. Entretanto, embora os estudantes do oitavo período apresentem maior média de horas para monitoria, apenas dois estudantes do oitavo período responderam a esse item, não sendo possível extrapolar essa informação para o grupo, pois essa atividade é predominante no quarto período, tendo em vista que os alunos têm mais atividades teóricas e de simulação do que os do oitavo período, que têm mais atividades em cenário prático de ensino na FPS.

Seis riscos foram planejados pelos pesquisadores de acordo com as medidas preconizadas pelo Ministério da Saúde/ OMS para identificação dos estudantes. Entretanto, nenhum dos participantes identificou todos esses riscos. Esse resultado pode demonstrar certa falta de interesse dos estudantes, por considerarem o cuidado à segurança do paciente como um aspecto a ser avaliado pelos profissionais de enfermagem, por exemplo. Na simulação, não houve diferença estatística significativa entre os estudantes do quarto e oitavo períodos nos itens planejados pelos pesquisadores. Isto mostra que a maior experiência prática dos estudantes do oitavo período não parece ter impactado positivamente seu desempenho na simulação realizada. Resultado semelhante foi encontrado em pesquisas que compararam estudantes de diferentes períodos do curso e internos com médicos recém-formados. Nenhum estudante identificou o risco relacionado à prevenção de lesões por pressão. Esse resultado baixo é semelhante ao do estudo que baseou esta pesquisa, realizado na Universidade de Chicago (EUA), que avaliou a habilidade de estudantes de Medicina em identificar erros na segurança do paciente, em que apenas $5 \%$ dos estudantes identificaram o risco relacionado à prevenção de lesões por pressão. Isto pode ocorrer pelo fato de os estudantes não pensarem nos riscos de longo prazo que o ambiente oferece ao paciente, e, sendo assim, a lesão por pressão passa despercebida.

De maneira geral, a identificação dos riscos pelos estudantes foi baixa. Apenas a administração segura de medicamentos obteve mais de $80 \%$ de identificação entre os riscos planejados pelos pesquisadores - resultado abaixo do obtido no estudo em que se baseou esta pesquisa. Por outro lado, este resultado corrobora outro estudo que avalia estudantes de Medicina e Enfermagem na identificação de riscos à segurança do paciente. Os demais riscos planejados - identificação do paciente, prevenção de quedas, higienização das mãos e cirurgia segura - não apresentaram diferença estatística entre os períodos dos estudantes.

Três riscos adicionais não planejados pelos pesquisadores foram identificados pelos estudantes que participaram da pesquisa: necessidade de isolamento dos pacientes, necessidade de uso de Equipamento de Proteção Individual (EPI) e distância entre os leitos dos pacientes. Esses riscos podem demonstrar a preocupação dos estudantes com a biossegurança e a necessidade de prevenção de doenças infectocontagiosas.

Entre os três riscos adicionais identificados e não planejados, houve diferença estatística significativa quanto à identificação do risco de isolamento dos pacientes, que apresentou uma média de acertos de mais de $50 \%$, sendo a identificação maior entre os estudantes do oitavo período, o que pode demonstrar maior conhecimento acerca das doenças dos casos da simulação. Essa média de acertos para o risco de isolamento foi um pouco maior que a encontrada em pesquisa norte-americana que avaliou o desempenho de estudantes de Enfermagem e Medicina para identificar erros na segurança do paciente. A distância entre os leitos dos pacientes foi identificada por $21 \%$ dos estudantes do quarto período e por nenhum estudante do oitavo período. Este resultado pode demonstrar a maior carga horária de aulas teóricas e de simulação que faz parte da matriz curricular do ciclo básico do curso médico em comparação com o ciclo clínico. Por fim, a necessidade do uso de EPI, embora apresente baixa média de acertos e não apresente diferença estatística, teve predomínio de identificação entre os estudantes do oitavo período. Este resultado condiz com o do estudo que baseou esta pesquisa e que apresentou maior identificação entre os estudantes mais adiantados no curso.

Este modelo de estudo é replicável em outros lugares em virtude da baixa complexidade dos recursos necessários e por se tratar de um modelo personalizável para o ambiente onde será adotado. Todavia, requer planejamento demorado e infraestrutura que permita uma situação o mais próxima possível do cenário real. O caso clínico aplicado no estudo foi 
de baixa complexidade e baseado nos principais riscos a que os pacientes estão sujeitos quando hospitalizados, segundo a OMS e o Ministério da Saúde. Os estudantes que optaram por receber feedback o receberam via e-mail, obtendo seu desempenho individual comparado com o geral. Entre as limitações deste estudo, está a não realização de uma simulação piloto para corrigir eventuais problemas na simulação desenvolvida, a grande perda de estudantes devido à indisponibilidade de horário dos alunos, a realização de apenas um dia de simulação e a não abordagem prévia do tema segurança do paciente ao longo da formação dos estudantes.

\section{CONCLUSÃO}

A segurança do paciente é um tema fundamental para os estudantes da área de saúde. Este estudo demonstra que, apesar de menos da metade dos estudantes participantes identificarem os riscos à SP - como não identificação do paciente, não prevenção de queda, falta de higienização das mãos, não realização de cirurgia segura, insuficiente distância entre os leitos e ausência de EPI -, houve ganho de competência, habilidades e atitudes na identificação de alguns desses riscos ao longo do curso médico, principalmente na identificação dos riscos administração segura de medicação e necessidade de isolamento.

Assim, concluímos que, embora os estudantes tenham identificado menos da metade dos riscos planejados pelos pesquisadores, houve ganho de conhecimentos, habilidades e atitudes ao longo da formação médica dos estudantes em identificar riscos à segurança do paciente.

\section{REFERÊNCIAS}

1. ANVISA, Agência Nacional de Vigilância Sanitária. Assistência segura: uma reflexão teórica aplicada à prática. Série: Segurança do Paciente e Qualidade em Serviços de Saúde.

2. Runciman W, Hibbert P, Thomson R, Schaaf TVD, Sherman $\mathrm{H}$, Lewalle P. Towards an International Classification for Patient Safety: key concepts and terms. InternationalJournal for Quality in Health Care. Feb 2009;21(1):18-26.

3. ANVISA, Agência Nacional de Vigilância Sanitária. Boletim Segurança do Paciente e Qualidade em Serviços de Saúde - Incidentes Relacionados à Assistência à Saúde - 2015.

4. IESS, Instituto de Estudos de Saúde Suplementar. Anuário da segurança assistencial hospitalar no Brasil.

5. CDC, Centers for Disease Control and prevention. Healthcare-associated Infections (HAI) Progress Report. 2016.

6. WHO, World Health Organization Health care-associated infections Fact Sheet. 2014.

7. Clay AS, Chudgar SM, Turner KM, Vaughn J, Knudsen NW, Farnan JM, Arora VM, Molloy MA. How Prepared Are Medical and Nursing Students to Identify Common
Hazards in the Intensive Care Unit? Ann Am Thorac Soc. 2017 Apr;14(4):543-549.

8. Joyce A Wahr, MD, FAHA. Operating room hazards and approaches to improve patient safety. Apr 11, 2018.

9. Ministério da Saúde (Brasil). Gabinete do Ministro. Portaria MS/GM n 529, de 1 de abril de 2013.

10. Bogarin, Denise Franze et al. Segurança do paciente: conhecimento de alunos de graduação em enfermagem. CogitareEnferm. 2014 Jul/Set; 19(3):491-7.

11. Laatikainen O, Miettunen J, Sneck S, Lehtiniemi H, Tenhunen $\mathrm{O}$, Turpeinen $\mathrm{M}$. The prevalence of medication-related adverse events in inpatients-a systematic review and meta-analysis. Eur J Clin Pharmacol. 2017 Dec;73(12):1539-1549.

12. Davenport MC, Domínguez PA, Ferreira JP, Kannemann AL, Paganini A, Torres FA. Measuring adverse events in pediatric inpatients with the Global Trigger Tool. Arch Argent Pediatr. 2017 Aug 1;115(4):357-363.

13. Agencia Nacional de Vigilância Sanitária (Brasil). Portaria no. 529, de 1 de abril de 2013. Institui o Programa Nacional de Segurança do Paciente (PNSP). Diário Oficial da União 2abr 2013; Seção 1.

14. World Health Organization (WHO). WHO World Alliance for Patient Safety. Forward Programme 2008-2009. Geneva: WHO, 2008.

15. Reeves SA, Denault D, Huntington JT, Ogrinc G, Southard DR, Vebell R. Learning to Overcome Hierarchical Pressures to Achieve Safer Patient Care: An Interprofessional Simulation for Nursing, Medical, and Physician Assistant Students. Nurse Educ. 2017 Sep/Oct;42(5S Suppl 1):S27-S31.

16. Daud-Gallotti RM, Morinaga CV, Arlindo-Rodrigues M, Velasco IT, Martins MA, Tiberio IC. A new method for the assessment of patient safety competencies during a medical school clerkship using an objective structured clinical examination. Clinics (Sao Paulo). 2011;66(7):1209-15.

17. Yoshikawa Jamile Mika, Sousa Bruna Elisa Catin de Peterlini Maria Angélica Sorgini, Kusahara Denise Miyuki, Pedreira Mavilde da Luz Gonçalves, Avelar Ariane Ferreira Machado. Compreensão de alunos de cursos de graduação em enfermagem e medicina sobre segurança do paciente. 26(1): 21-29.

18. Panagioti M, Stokes J, Esmail A, Coventry P, Cheraghi-Sohi S, Alam R, Et al. Multimorbidity and Patient Safety Incidents in Primary Care: A Systematic Review and Meta-Analysis. PLoSOne. 2015 Aug 28;10(8): e0135947.

19. Rubin G, George A, Chinn DJ, Richardson C. Errors in general practice: development of an error classification and pilot study of a method for detecting errors. QualSaf Health Care. 2003; 12(6):443-7.

20. Dearden E, Mellanby E, Cameron H, Harden J. Which non-technical skills do junior doctors require to prescri- 
be safely? A systematic review. Br J ClinPharmacol. 2015 Dec;80(6):1303-14.

21. Escher C, Creutzfeldt J, Meurling L, Hedman L, Kjellin A, Felländer-Tsai L. Medical students' situational motivation to participate in simulation-based team training is predicted by attitudes to patient safety. BMC Med Educ. $2017 \mathrm{Feb}$ 10;17(1):37.

22. Granheim BM, Shaw JM, Mansah M. The use of interprofessional learning and simulation in undergraduate nursing programs to address interprofessional communication and collaboration: An integrative review of the literature. Nurse Educ Today. 2018 Mar; 62:118-127.

23. Brasil. Diretrizes Curriculares Nacionais do Curso de Graduação em Medicina. Resolução № 3, de 20 de junho de 2014.

24. Bohomol Elena, Freitas Maria Aparecida de Oliveira, Cunha Isabel Cristina KowalOlm. Ensino da segurança do paciente na graduação em saúde: reflexões sobre saberes e fazeres; 20( 58): 727-741.

25. BOHOMOL, Elena; KOWAL OLM CUNHA, Isabel Cristina. Ensino sobre segurança do paciente no curso de Medicina da Universidade Federal de São Paulo. Einstein (16794508), v. 13, n. 1, 2015.

26. Teagle AR, George M, Gainsborough N, Haq I, Okorie M. Preparing medical students for clinical practice: easing the transition. Perspect Med Educ. 2017 Aug;6(4):277-280.

27. Crimmins AC, Wong AH, Bonz JW, Tsyrulnik A, Jubanyik K, Dziura JD, Dodge KL, Evans LV. “To Err Is Human” but Disclosure Must be Taught: A Simulation-BasedAssessment Study. Simul Healthc. 2018 Apr;13(2):107-116.

28. Pontifícia Universidade Católica do Rio de Janeiro - PUC-Rio. Guia Curricular de Segurança do Paciente da Organização Mundial da Saúde: Edição Multiprofissional.

29. Farnan JM, Gaffney S, Poston JT, et al. Patient safety room of horrors: a novel method to assess medical students and entering residents' ability to identify hazards of hospitalization. BMJ Qual Saf 2016; 25:153-158.

30. BRANDÃO, C. F.; COLLARES, Carlos Fernando; MARIN, H. F. A simulação realística como ferramenta educacional para estudantes de medicina. Sci Med, v. 24, n. 2, p. 187-92, 2014.

31. Ministério da Saúde (BR). Fundação Oswldo Cruz (Fiocruz). Agência Nacional de Vigilância Sanitária (ANVISA). Documento de referência para o Programa Nacional de Segurança do Paciente.

32. Karoline Pedroti Fiorotti et al. Perfil do Estudante de Medicina da Universidade Federal do Espírito Santo. Revista Brasileira de Educação Médica 34 (3): 355-362; 2010.
33. Lamounier JA, Torga ALGF, Leite GLF. Características e conceitos dos formandos do Curso de Medicina da UFMG. Pediatria (São Paulo). 2002; 24(3/4):85-92.

34. Dhalla IA, Kwong JC, Streiner DL, Baddour RE, Waddell $\mathrm{AE}$, Johnson IL. Characteristics of first-year students in Canadian medical schools. CMAJ. 2002;166(8):1029-35.

35. Márcia Miki Tanaka et al. Adaptação de alunos de medicina em anos Iniciais da Formação. Revista Brasileira de Educação Médica. 40 (4): 663-668; 2016.

36. Mattos P, Nazar BP, Tannock R. By the book: ADHD prevalence in medical students varies with analogous methods of addressing DSM items. Rev Bras Psiquiatr. 2018 Feb 15:0.

37. Pacheco JP, Giacomin HT, Tam WW, Ribeiro TB, Arab C, Bezerra IM, Pinasco GC. Mental health problems among medical students in Brazil: a systematic review and meta-analysis. Rev Bras Psiquiatr. 2017 Oct-Dec;39(4):369-378.

38. Instituto de Pesquisa Econômica Aplicada. Estudo Comparado sobre a Juventude Brasileira e Chinesa. 2017.

39. José Antonio Chehuen Neto et al. Fontes de estudo e pesquisa entre os estudantes de medicina. Rev Med Minas Gerais 2016; 26: e-1787.

40. Morgan J, Green V, Blair J. Using simulation to prepare for clinical practice. Clin Teach. 2018 Feb;15(1):57-61.

\section{CONTRIBUIÇÃO DOS AUTORES}

Carlos Vinícius Pacheco dos Santos Guaraná: contribuiu na concepção e delineamento da pesquisa, na análise e interpretação dos dados, na redação do manuscrito, na revisão crítica e na aprovação final. Edvaldo da Silva Souza: contribuiu na concepção e delineamento da pesquisa, na análise e interpretação dos dados, na redação do manuscrito, na revisão crítica e na aprovação final. Victor de Souza Dias: contribuiu na concepção e delineamento da pesquisa, na coleta, análise e interpretação dos dados e na redação do manuscrito. Eliana Valentim: contribuiu na concepção e delineamento da pesquisa e na coleta de dados.

\section{CONFLITO DE INTERESSES}

Declaramos não haver conflitos de interesse.

\section{ENDEREÇO PARA CORRESPONDÊNCIA}

Avenida Mal. Mascarenhas de Morais, 4861, Imbiribeira, Recife-PE

CEP: $51.150-000$

Telefone: (81) 3035.7777 / (81) 3312.7777 